\title{
SUR LA CONTINUITÉ AUTOMATIQUE DES ÉPIMORPHISMES DANS LES $\star$-ALGĖBRES DE BANACH
}

\author{
L. OUKHTITE, A. TAJMOUATI, and Y. TIDLI
}

Reçu le 26 avril 2001 et en forme révisée le 18 janvier 2002

\begin{abstract}
Nous étudions les problèmes de continuité automatique dans des algèbres de Banach avec involutions. Nous obtenons aussi des nouveoux résultats concernant $\star$-idéals des $\star$-algèbres.
\end{abstract}

Classification 2000 des Sujets Mathématiques: 46J10, 46K05.

1. Préliminaires. La majorité des définitions et des résultats qui sont rappelés ici se trouvent dans [1]. Les algèbres considérées sont supposées sur $\mathbb{C}$, unitaires, non nécessairement commutatives.

Une involution sur une algèbre $A$ est une application $\star: A \rightarrow A$ vérifiant les propriétés suivantes :

$$
\begin{gathered}
\left(x^{\star}\right)^{\star}=x, \quad(x+y)^{\star}=x^{\star}+y^{\star}, \quad(x y)^{\star}=y^{\star} x^{\star}, \quad(\lambda x)^{\star}=\bar{\lambda} x^{\star}, \\
\forall x, y \in A, \forall \lambda \in \mathbb{C} .
\end{gathered}
$$

Munie de l'involution $\star, A$ est dite une $\star$-algèbre. Une involution $\star$ est dite anisotrope si pour tout $a$ dans $A$ on a : $a^{\star} a=0 \Rightarrow a=0$. Un idéal $I$ d'une $\star$-algèbre est dit $\star$-idéal si $I^{\star} \subset I$ (et alors $I^{\star}=I$ ). Il en résulte alors que tout $\star$-idéal est bilatère. De plus, si les seuls $\star$-idéaux de $A$ contenu dans $I$ sont $(0)$ et $I$ alors on dit que $I$ est $\star$-minimal. Remarquons que si $I$ est un $\star$-idéal non nul de $A$, alors $\star$ induit une involution sur $A / I$, notée aussi $\star$, définie par : $(a+I)^{\star}=a^{\star}+I$. Un $\star$-idéal $\mathcal{M}$ est dit $\star$-maximal si les seuls $\star$-idéaux contenant $M$ sont $A$ et $M$. Une algèbre $A$ est dite simple si les seuls idéaux bilatères de $A$ sont ( 0 ) et $A$. Dans le cas où $A$ admet une involution $\star$, on dira que $A$ est $\star$-simple si les seules $\star$-idéaux de $A$ sont (0) et $A$.

Remarquons que si $A$ est une algèbre simple munie d'une involution $\star$, alors $A$ est

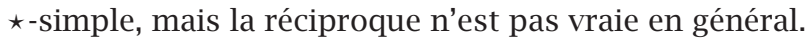

EXEMPLE 1.1. Soit $A$ une algèbre simple et $A^{\circ}$ l'algèbre opposée de $A$. Considérons alors l'algèbre $B=A \times A^{\circ}$, munie de l'involution d'échange $\star$ définie par : $\star(x, y)=$ $(y, x)$. Alors une simple vérification montre que $B$ est une algèbre $\star$-simple mais n'est pas simple.

Un idéal $P$ de $A$ est $\star$-premier (resp. $\star$-semi-premier) si pour deux $\star$-idéaux $I$ et $J$ de $A$ tels que $I J \subseteq P$ (resp. $I^{2} \subseteq P$ ) alors $I \subseteq P$ ou $J \subseteq P$ (resp. $I \subseteq P$ ). En outre, si (0) est $\star$-premier (resp. $\star$-semi-premier) on dit que $A$ est $\star$-première (resp. $\star$-semi-première). 
Soit $I$ un idéal minimal à gauche d'une algèbre semi-première $A$, alors il existe un idempotent minimal $e \in A$ tel que $I=A e$.

Rappelons que le radical de Jacobson, $\operatorname{Rad}(A)$, d'une algèbre $A$ est définit comme étant l'intersection de tous les idéaux à gauche maximaux de $A$. Si de plus $A$ est une $\star$-algèbre, alors le $\star$-radical de $A$, noté $\operatorname{Rad}_{\star}(A)$, est l'intersection de tous les idéaux $\star$-maximaux de $A$. En outre, si $\operatorname{Rad}_{\star}(A)=(0)$ alors $A$ est dite $\star$-semi-simple.

Soit $T$ une application linéaire d'un espace de Banach $X$ dans un espace de Banach $Y$. Alors l'espace séparateur $\sigma(T)$ de $T$ est un sous-espace de $Y$ défini par:

$$
\sigma(T)=\left\{y \in Y \mid \exists\left(x_{n}\right)_{n} \in X: x_{n} \underset{n \rightarrow \infty}{\longrightarrow} 0 \text { et } T\left(X_{n}\right) \underset{n \rightarrow \infty}{\longrightarrow} y\right\} .
$$

Il est bien connu que $T$ est continue si, et seulement si, $\sigma(T)=(0)$ [2]. En outre, l'espace séparateur d'un épimorphisme d'une algèbre de Banach $A$ dans une algèbre de Banach $B$ est un idéal bilatère fermé [2]. De plus, si $\theta$ est un épimorphisme d'une algèbre de Banach $A$ sur une algèbre de Banach $B$ et si $b \in \sigma(\theta)$, alors $o \in \operatorname{Sp}(b)$ [2, théorème 6-16].

2. La continuité automatique dans une algèbre de Banach $\star$-simple. Nous commençons par donner quelques propositions préliminaires utiles pour la suite.

Proposition 2.1. Soit I un idéal $\star$-minimal d'une $\star$-algèbre A. Si I n'est pas minimal et si $J$ est un idéal inclu strictement dans $I$, alors $I=J \oplus J^{\star}$, où $J$ et $J^{\star}$ sont des idéaux minimaux de A. Si de plus $I^{2} \neq 0$, alors $J$ et $J^{\star}$ sont les seuls idéaux non nuls contenu strictement dans I.

Démonstration. Supposons que $I$ n'est pas minimal et soit $J$ un idéal non nul de $A$ contenu strictement dans $I$. On a $J+J^{\star}$ et $J \cap J^{\star}$ sont deux $\star$-idéaux de $A$ contenus dans $I$. Or $I$ est un idéal $\star$-minimal, donc $J \cap J^{\star}=(0)$ et $J+J^{\star}=I$. Par conséquence, $I=J \oplus J^{\star}$. Soit $Y$ un idéal non nul de $A$ tel que $Y \subset J$. Un raisonnement analogue montre que : $I=Y \oplus Y^{\star}$. De plus, si $k \in J$ alors $k=y_{1}+y_{2}$, où $y_{1} \in Y$ et $y_{2} \in Y^{\star}$. Par suite $k-y_{1}=y_{2} \in J \cap J^{\star}=(0)$, donc $k \in Y$ de sorte que $J=Y$. Par conséquent, $J$ (resp. $J^{\star}$ ) est un idéal minimal de $A$.

Supposons maintenant que $I^{2} \neq 0$ et soit $B$ un idéal non nul de $A$ tel que $B \subset I$, $B \neq J$ et $B \neq J^{\star}$. Une simple vérification montre que $B$ est un idéal minimal de $A$. Par conséquence $B J=(0)$, de même on trouve que $B^{\star} J=B J^{\star}=B^{\star} J^{\star}=(0)$. D'où $I^{2}=\left(B \oplus B^{\star}\right)\left(J \oplus J^{\star}\right)=(0)$, ce qui contredit le fait que $I^{2} \neq(0)$.

Proposition 2.2. Soient $I_{1}$ et $I_{2}$ deux idéaux d'une $\star$-algèbre A tels que : $A=I_{1} \oplus I_{2}$ et $I_{2}=I_{1}^{\star}$. Si $I_{1}$ et $I_{2}$ sont minimaux alors A est une algèbre $\star$-simple.

Démonstration. On a $A^{2}=\left(I_{1} \oplus I_{2}\right)\left(I_{1} \oplus I_{2}\right)=I_{1}^{2} \oplus I_{1} I_{2} \oplus I_{2} I_{1} \oplus I_{2}^{2}$. Comme $I_{1} I_{2}$ et $I_{2} I_{1}$ sont inclus dans $I_{1} \cap I_{2}=(0)$, on en déduit que $A^{2}=I_{1}^{2} \oplus I_{2}^{2}$. Or $I_{1}^{2} \subset I_{1}$ et $I_{2}^{2} \subset I_{2}$, le fait que $I_{1}$ et $I_{2}$ sont minimaux donne alors $I_{1}^{2}=(0)$ ou $I_{1}^{2}=I_{1}$. Si $I_{1}^{2}=(0)$ alors $I_{2}^{2}=(0)$, par suite $A^{2}=(0)$. Ce qui est impossible puisque $A$ est unitaire. Donc $I_{1}^{2}=I_{1}$ de sorte que $I_{2}^{2}=I_{2}$. D'où $A^{2}=A$, dans ce cas les seuls $\star$-idéaux de $A$ sont (0) et $A$. Par conséquence, $A$ est une algèbre $\star$-simple. 
Proposition 2.3. Pour une $\star$-algèbre A, soient les assertions suivantes :

(i) A est $\star$-simple

(ii) A est $\star$-première

(iii) A est $\star$-semi-première

(iv) A est semi-première.

Alors on a $(\mathrm{i}) \Rightarrow(\mathrm{ii}) \Rightarrow(\mathrm{iii}) \Rightarrow($ iv $)$.

Démonstration. (i) $\Rightarrow$ (ii) Soient $I$ et $J$ deux $\star$-idéaux de $A$ non nuls, alors $I J$ est non nuls, car $I J=A^{2} \neq(0)$. Par conséquent, $A$ est $\star$-première.

(ii) $\Rightarrow$ (iii) Evident.

(iii) $\Rightarrow$ (iv) Soit $I$ un idéal à gauche de $A$ tel que $I^{2}=(0)$. Alors $\left(I \cap I^{\star}\right)^{2}=(0)$, ce qui implique que $I \cap I^{\star}=(0)$. D'autre part, on a

$$
\left(I+I^{\star}\right)^{2}=I^{2}+I I^{\star}+I^{\star} I+\left(I^{\star}\right)^{2}=\left(I^{\star}\right)^{2}=\left(I^{2}\right)^{\star}=(0) .
$$

Par conséquent, $I \subseteq I+I^{\star}=(0)$.

Proposition 2.4. Soit A une algèbre de Banach simple unitaire d'unité e. Alors tout épimorphisme d'une algèbre de Banach sur A est continu.

Démonstration. Soit $\theta: B \rightarrow A$ un épimorphisme d'une algèbre de Banach $B$ sur $A$ et soit $e$ l'unité de $A$. Le fait que $\sigma(\theta)$ un idéal de $A$, entraîne alors que $\sigma(\theta)=(0)$ ou $\sigma(\theta)=A$. Si $\sigma(\theta)=A$ alors $e \in \sigma(\theta)$, par suite $0 \in \operatorname{Sp}(e)$ [2, théorème 6-16], ce qui est absurde. Donc $\sigma(\theta)=(0)$, par conséquent $\theta$ est continu.

THÉORÈme 2.5. Soient A une algèbre de Banach et B une algèbre de Banach $\star$-simple. Alors tout épimorphisme de A dans B est continu.

Démonstration. Soit $\theta: A \rightarrow B$ un épimorphisme. Si $B$ est une simple algèbre, d'après la proposition 2.4, $\theta$ est automatiquement continu. Si $B$ n'est pas simple, alors $B$ est somme directe de deux sous-algèbres simples. En effet, comme $B$ est $\star$-simple qui n'est pas simple, on peut considérer $B$ comme un idéal $\star$-minimal qui n'est pas minimal dans lui même. Donc, pour tout idéal non nul propre $J$ de $B$, on a $B=J \oplus J^{\star}$, avec $J$ et $J^{\star}$ sont des idéaux minimaux de $B$ (voir proposition 2.1).

Montrons que $J$ est une algèbre simple. Soit donc $T$ un idéal non nul de $J$, alors $T$ est un idéal de $B$. En effet, soit $b$ un élément de $B$ et $t$ un élément de $T$, alors il existe $j$ et $j^{\prime}$ deux éléments de $J$ tels que $b=j+j^{\prime *}$, d'où $b t=\left(j+j^{\prime *}\right) t=j t+j^{\prime *} t$. Puisque $j^{*} t \in J \cap J^{\star}=\{0\}$, alors $b t=j t \in T$. Ce qui implique que $T$ est un idéal de $A$. D’après la minimalité de $J$, nécessairement $T=J$, (même chose pour $J^{\star}$ ). En outre, $B$ est semi-première (proposition 2.3), alors il existe un idempotent $e \in B$ tel que $J=B e$ et $J^{\star}=B e^{\star}$. Donc $J$ (resp. $J^{\star}$ ) est une sous-algèbre unitaire d'unité $e$ (resp. $e^{\star}$ ). De plus, on a $B / J^{\star} \simeq J$ et puisque $J^{\star}$ est minimal alors $J$ est un idéal maximal de $B$. Un raisonnement analogue montre que $J^{\star}$ est aussi un idéal maximal de $B$. Comme $B$ est une algèbre de Banach, alors $J$ est un idéal fermé [2, lemme 6-3]. Par conséquence, munie de la norme induite, $J$ (resp. $J^{\star}$ ) est une algèbre de Banach simple. De plus, si $\operatorname{Pr}_{1}$ (resp. $\operatorname{Pr}_{2}$ ) désigne la projection canonique de $B$ sur $J$, (resp. de $B$ sur $J^{\star}$ ), alors d'après 
la proposition précédente $\operatorname{Pr}_{1} \circ \theta$ (resp. $\operatorname{Pr}_{2} \circ \theta$ ) est continue. Par suite $\operatorname{Pr}_{1} \circ \theta+\operatorname{Pr}_{2} \circ \theta=\theta$ est continu.

Corollaire 2.6. Soit B une algèbre de Banach $\star$-simple. Alors B possède une unique norme d'algèbre de Banach.

DÉmonstration. Il suffit d'appliquer le théorème précédent à l'identité de $B$.

REMARQUe 2.7. Si $A$ est une algèbre $\star$-simple et si l'involution $\star$ est anisotrope, alors $A$ est simple.

Proposition 2.8. Soient $A$ une $\star$-algèbre et $M$ un idéal $\star$-maximal qui n'est pas maximal de A. Alors il existe un idéal maximal $N$ tel que $N+N^{\star}=$ A et $N \cap N^{\star}=M$.

Démonstration. Puisque $A / M$ est une algèbre $\star$-simple qui n'est pas simple, la proposition 2.1 assure l'existence d'un idéal propre $N$ de $A$ avec $M \subset N$ tel que $A / M=$ $(N / M) \oplus(N / M)^{\star}$, où $N / M$ et $(N / M)^{\star} \simeq N^{\star} / M$ sont deux idéaux minimaux et maximaux à la fois de $A / M$. Par conséquence, $N$ et $N^{\star}$ seront deux idéaux maximaux de $A$ vérifiant $N+N^{\star}=A$ et $N \cap N^{\star}=M$.

COROLLAIRE 2.9. Toute algèbre «-semi-simple est semi-simple.

DÉmonstration. Soit $A$ une algèbre $\star$-semi-simple, alors $\operatorname{Rad}_{\star}(A)=\bigcap M=0$, où $\bigcap M$ désigne l'intersection de tous les idéaux $\star$-maximaux de $A$. Mais $M=N \cap N^{\star}$ par la proposition 2.8, où $N$ est un idéal maximal de $A$. En outre, si $N$ est un idéal maximal alors $N^{\star}$ est aussi un idéal maximal. Donc $\bigcap_{M \star-m a x i m a l} M \supseteq \bigcap_{N \text { maximal }}\left(N \cap N^{\star}\right)$, le fait que

$$
\operatorname{Rad}(A) \subseteq \bigcap_{M \text { maximal }} M=\bigcap_{N \text { maximal }}\left(N \cap N^{\star}\right) \subseteq \bigcap_{M \star-\text { maximal }} M=\operatorname{Rad}_{\star}(A)=0
$$

donne alors que $A$ est semi-simple.

Proposition 2.10. Soient $A$ une $\star$-algèbre de Banach et $M$ un idéal $\star$-maximal de A. Alors $M$ est fermé dans A.

Démonstration. Si $M$ est un idéal maximal, alors $M$ est fermé. Si $M$ n'est pas maximal, la proposition précédente entraîne l'existence d'un idéal maximal $N$ tel que $N \cap N^{\star}=M$. Comme $N$ et $N^{\star}$ sont deux ideal fermés, alors $M$ est aussi un idéal fermé.

Corollaire 2.11. Soit $M$ un idéal $\star$-maximal d'une $\star$-algèbre de Banach A. Alors A/M est une algèbre de Banach $\star$-simple.

Les résultats suivants sont des conséquences du corollaire 2.9.

THÉORÈmE 2.12. Soit B une algèbre de Banach $\star$-semi-simple. Alors :

(1) tout épimorphisme d'une algèbre de Banach dans $B$ est continu;

(2) toutes les normes complètes sur $B$ sont équivalentes;

(3) l'involution $\star$ est automatiquement continue sur $B$. 


\section{Bibliographie}

[1] L. H. Rowen, Ring Theory. Vol. I, Pure and Applied Mathematics, vol. 127, Academic Press, Massachusetts, 1988.

[2] A. M. Sinclair, Automatic Continuity of Linear Operators, Cambridge University Press, Cambridge, 1976.

L. Oukhtite : Département de Mathématiques et Informatique, Faculté des Sciences DharMahraz, B.P 1796 Atlas Fès, Morocco

E-mail address: oukhtite@caramai1.com

A. Tajmouati : Département de Mathématiques et Informatique, Faculté des Sciences DharMahraz, B.P 1796 Atlas Fès, Morocco

E-mail address: atajmouati@yahoo.fr

Y. Tidli : Département de Mathématiques et Informatique, Faculté des Sciences Dhar-Mahraz, B.P 1796 Atlas Fès, Morocco

E-mail address: ytid7i@math. net 


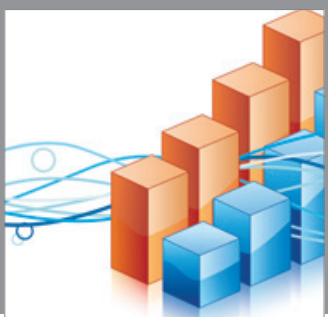

Advances in

Operations Research

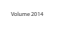

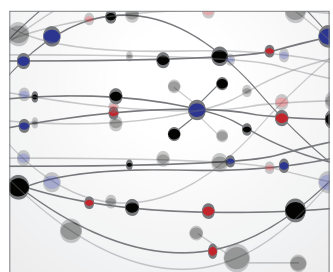

\section{The Scientific} World Journal
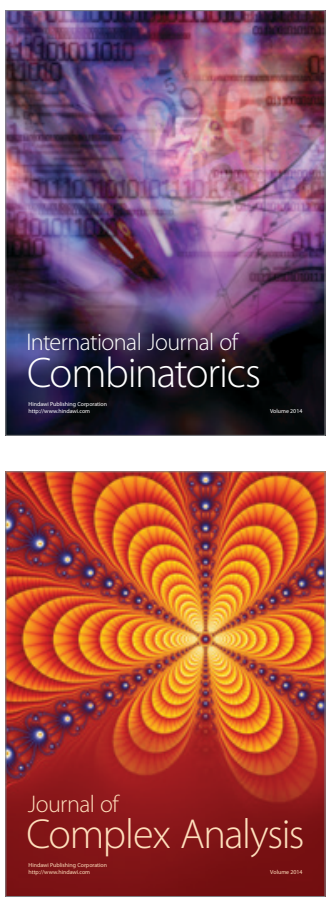

International Journal of

Mathematics and

Mathematical

Sciences
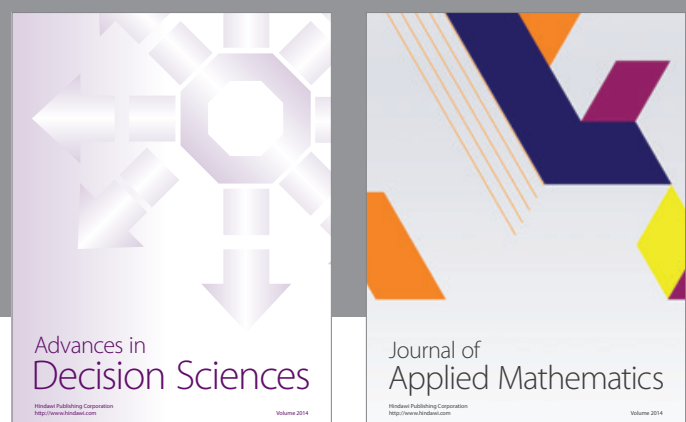

Journal of

Applied Mathematics
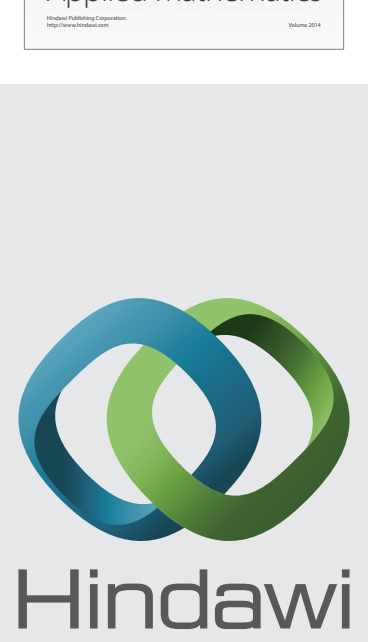

Submit your manuscripts at http://www.hindawi.com
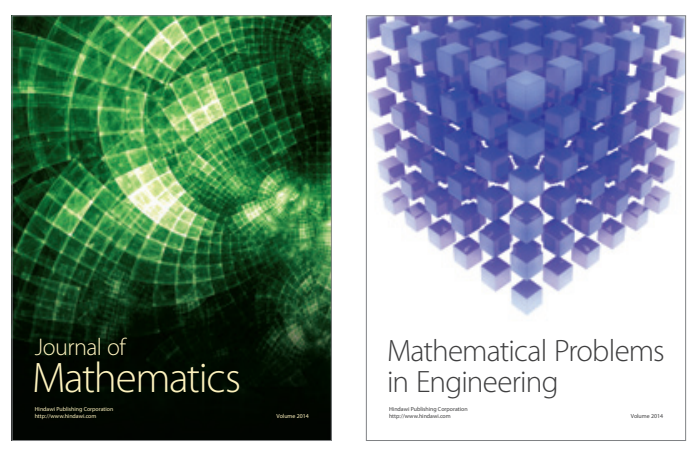

Mathematical Problems in Engineering
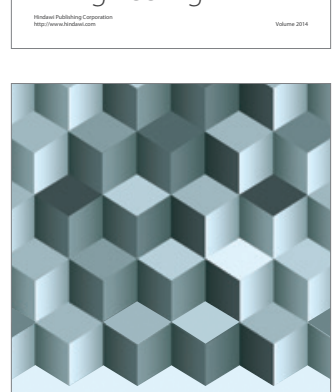

Journal of

Function Spaces
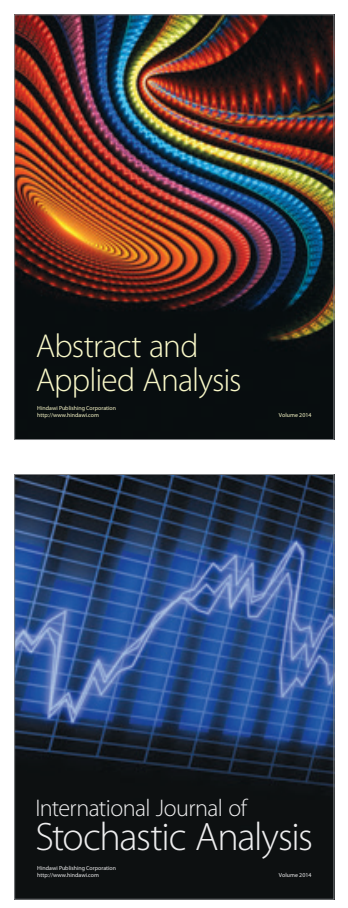

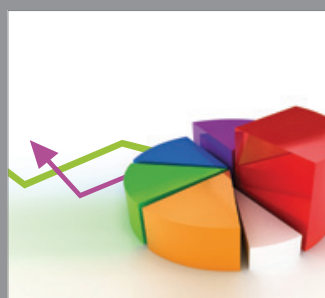

ournal of

Probability and Statistics

Promensencen
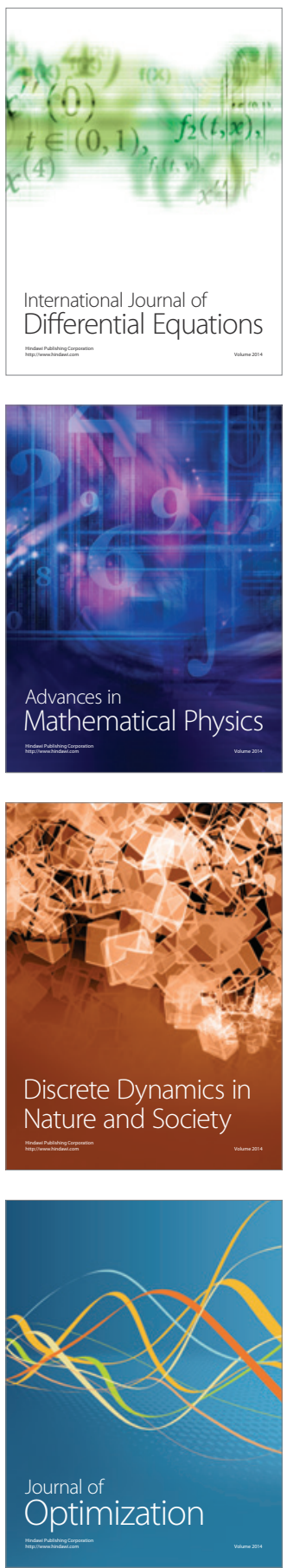